\title{
List of Reviewers 2012
}

Eastern Economic Journal (2013) 39, 283. doi:10.1057/eej.2013.8

The Editors and Editorial Board wish to acknowledge the assistance of the following colleagues, who reviewed articles during 2012, for their contributions of time and experience. Without their dedication, our work in publishing this journal could not go forward.

Peter von Allmen
D. Mark
Anderson
Ola Andersson
Laura Argys
Susan Averett
Cynthia Bansak
Silvia Barcellos
Timothy Bates
Reagan Baughman
Nicole Bissessar
Howard Bodenhorn
John Bonin
Jason Brown
Thomas Bruggink
Ronald Caldwell, Jr
Santiago
Carbo-Valverde
Geoffrey Carliner
Kelly Carter
Carolina Castilla
Satya Chakravarty
Donald Chambers
Trevor Collier
Karen Conway
Hope Corman
Terry-Ann Craigie
Claire Crutchley
Jeff Cummins
Dhaval Dave
Lewis Davis
Nicolas de Roos
James DeVault
Lisa Dickson
Angela Dills

Andrew
Economopoulos
Eric Eide
Glenn Ellison
Sarah Estelle
Fadi Fawaz
Edward Feasel
Nicholas Feltovich
Angela Fertig
Sean Flaherty
Erin Fletcher
Peter Francia
Michael French
Connel Fullenkamp
Winnie Fung
Jesus Garcia-Iglesias
Isabel-Maria
Garcia-Sanchez
Christopher Gore
Marshall Graham
Kent Grote
Federico Gutierrez
George Hall
Stephen Happel
James Hartley
Jerome Heavey
Rey Hernández-Julián
Phillip Hersh
Matthew Hill
Brett Hollenbeck
Paul Holmes
Fung-Mey Huang
Sandra Huston
Matthew Jaremski
Stephen Jenkins

Jaewon Jung

Michail Karoglou

Michael Kelly

Thomas Kinnaman

Jennifer Kohn

David Laband

Jonathan Lafky

Joanna Lahey

Leigh Ann Leung

Sarah Lyon

Mark Manfredo

Michael Marlow

Victor Matheson

Amalia Miller

Stephen Miller

Leslie Muller

Matthew Nagler

George Naufal

Rebecca Neumann

Farrokh Nourzad

Ilan Noy

Richard Nyuur

Anna Owen

Rodney Paul

Rafaela

Pizarro-Barcelo

Anne Polivka

James Poterba

Ioannis Pragidis

Andrea Presbitero

Agnes Quisumbing

Rati Ram

Justin Rao

Alicia Robb

Kurt Rotthoff
Peter Rutland

Scott Savage

Ondrej Schneider

Bruce Seaman

Mark Setterfield

Mark Showalter

Elias Shukralla

Asia Sikora

Curtis Simon

Julie Smith

Karen Smith

Chad Sparber

Herman O. Stekler

David Stifel

James Sundali

Ayca Tekin-Koru

Jason Thomas

Roy Thurik

Lise Tole

Adrian Tschoegl

Francesco Vona

Gary Wagner

Daniel Waldenstrom

Douglas Walker

Justin Wang

Yang Wang

Christian Weller

Roger White

Kate Williams

Nicholas Wilson

Paul Winters

Emilio Zanetti Chini

Madeline Zavodny

William Ziemba 